\title{
El intermedio y sus tiempos. Formas de circulación e impacto local de la invasión de México en el Pacífico Sur. Perú, Bolivia y Chile
}

The Intermission and its Times. Forms of Circulation and Local Impact of the Invasion of Mexico in the South Pacific. Peru, Bolivia and Chile

Matías Sánchez Barberán Ecole des Hautes Études en Sciences Sociales - Centre de Recherches sur les Mondes Américains, Francia mibarberan@hotmail.com

\section{Resumen}

Este artículo aborda las modalidades de circulación y el impacto de la invasión de México en el Pacífico Sur. Poniendo el acento en las formas y tiempos de circulación y en la calidad de la información, defiende la idea según la cual la masa crítica de informaciones transita de un conjunto de argumentos dispares a la convicción que hace de México la punta de lanza de la defensa republicana en el Nuevo Mundo. Esta modificación tendencial de la opinión es la base sobre la que se articula la respuesta sud-pacífica. Su virulencia se debe a la persistencia, en ambos bordes del Atlántico, de un pensamiento político que ve en la monarquía el régimen político idóneo para el continente. Realizando estas transformaciones, el Pacífico-Sur se muestra capaz de comprender los riesgos monárquicos a la luz de sus propios debates locales. La cuestión mexicana muestra sí los trazos de una historia común.

Palabras claves: Circulaciones; Pacífico Sur; República; Monarquía; opinión pública.

\begin{abstract}
This article addresses the modes of circulation and the impact of the invasion of Mexico in the South Pacific. Emphasizing the forms and times of circulation and the quality of information, it defends the idea according to which the critical mass of informations moves from a set of disparate arguments to the conviction that makes Mexico the spearhead of the Republican defense in the New World. This trend change of opinion in the basis on which the South Pacific reponse is articuled. Its
\end{abstract}

\section{Matías Sánchez Barberán}

El intermedio y sus tiempos. Formas de circulación e impacto local de la invasión de México en el Pacífico Sur. Perú, Bolivia y Chile.

Sur y Tiempo. Revista de Historia de América, №1, enero-junio 2020, pp. 47-72.

ISSN 2452-574X

DOI: $10.22370 /$ syt.2020.1.2046 
virulence is due to the persistence, on both sides of the Atlantic, of a political thought that sees in the monarchy the ideal political regime for the continent. By carriyng out these transformations the South Pacific is able to understand the monarchical risk in the light of its own local debates. The Mexican question does show the traces of a common history.

Keywords: Circulations; South Pacific; Republic; Monarchy; public opinion.

\section{Introducción}

Las tensiones de los años 1860 vuelven a poner al Nuevo Mundo en el centro de la atención. La Guerra de Secesión, la anexión de Santo Domingo a España, la expedición de México y la guerra entre España y las repúblicas del Pacifico-Sur son algunos de los conflictos que caracterizan este retorno (Palacios y Pani, 2014). Junto a la Guerra de Secesión, la expedición de México es uno de los que más conmociona al mundo occidental, según testimonios de los propios contemporáneos (Duchesne, 1976; Medzibrodsky, 1980; López, 1969; Lacoste, 1997). Desde Londres, Marx suspende la redacción del Capital para volverse hacia la cuestión mexicana, que define como "una de las más aberrantes empresas que se hayan consignado en los anales de la Historia internacional" (Marx, 2002: 426). Por su parte, John Russel, Ministro de Relaciones Exteriores Británico, alerta sobre una eventual guerra en el atlántico, que vendría alterar el orden geopolítico1. En Panamá, el corresponsal del Comercio de Lima resume en estos términos la irrupción de México en las tensiones atlánticas: "Mejico [...] se hace cada día más interesante por la aliada expedición que ha ido a visitar sus puertos"2. En La Paz, los lectores leen en El Telégrafo la misma conclusión: "la cuestión más importante del día es la [...] resolución a cerca de Méjico"3. El Ferrocarril no hace otra cosa, "el suceso que sigue llamando más la atención en Europa es sin duda la espedición a Méjico"4. De un diario al otro, la cuestión mexicana gana espacio en las columnas y los editoriales, pero también en las cartas privadas y la correspondencia diplomática.

Mirado más de cerca, la fuerza con la que México irrumpe en la agenda política entra en tensión con las vagas referencias que se tiene del país en el Pacifico-

1 John Russel à Charles Wyke, s/l (quizás Londres) 29 junio 1862. National Archives, PR030/22/95, $\mathrm{f}^{\circ} 25$.

2 El Comercio, 18 enero 1862.

3 El Telégrafo, 18 diciembre 1861, citado del Comercio.

4 El Eco Hispano-americano, reproducido en El Ferrocarril, 15 enero de 1862. Ver también El Ferrocarril, 24 de diciembre 1861. 
Sur. La conmoción y el desconcierto se agravan con la necesidad de conocer las reales intenciones de la expedición y la capacidad de respuesta del gobierno de Juárez. Necesidad satisfecha a condición de recurrir a la acción de numerosos intermediarios, que modifican irremediablemente la información en el curso de su transmisión (Slauter, 2012: 363-389; Kalifa et al., 2011; Walker, 2001). Esta característica puede parecer paradojal en un momento en que, al contrario, los escritos transmiten todo cuanto pueda dar una idea del nuevo escenario político, en un periodo que, como lo recuerda Elías José Palti (2005), la prensa conoce una inclinación profunda hacia la opinión en detrimento de las solas órdenes del poder. Los intercambios se multiplican con la precipitación de los hechos, mientras que la posibilidad de un retorno a la monarquía provoca una verdadera frenesís de la información (Basadre, 1846; Collier, 2005; Vaillant, 2011; 2017). La irrupción de México en la agenda política conduce a interrogar las formas de circulación de la información, así como su alteración, o no, durante esos desplazamientos.

Una perspectiva material y social de la circulación de información impone por un lado asociar espacios, objetos y formas de pensar la actualidad. Por otra parte, interrogar el impacto exterior al terreno inmediato del conflicto no es una pregunta nueva. La solidaridad hacia el México republicano es hoy un lugar común de la historiografía. La operación implica una jerarquización implícita del espacio, que conduce a pensar las reacciones ocurridas fuera del perímetro del conflicto como secundarias o marginales para el desenlace de la expedición. No se trata sin embargo de pensar la vinculación del Pacifico-Sur a México como un epifenómeno, ni menos en términos de influencia (Santana y Guerra, 2006). El objetivo es más bien observar las modalidades en que la cuestión de México pone en tensión las formas concretas de circulación, la demanda repentina de información y la inserción en los debates políticos locales. El presente artículo propone revistar estas cuestiones. Dos aspectos retienen la atención. Por un lado, las formas de circulación y la recepción en el Pacífico-Sur de las noticias sobre México. Por otra parte, la forma en que la cuestión mexicana modifica los debates locales a la luz de la interrogación sobre las implicaciones globales para el hemisferio. El argumento conduce a la hipótesis siguiente: la reacción del Pacífico-Sur a la cuestión mexicana reside en dos aspectos centrales. Por un lado, en la poca fiabilidad de la información, originada por la amplitud de las circulaciones y la indeterminación propia de la expedición. Por otro, más profundo y menos conocido, en la persistencia de corrientes que ven con simpatías la posibilidad de restablecer la monarquía en el Nuevo Mundo.

Dos tipos de análisis convergen en esta perspectiva. Por una parte, el acento en las condiciones externas de la circulación de la información permitirá evaluar su posibilidades materiales y técnicas. Por otra parte, la interrogación sobre la 
ambigüedad de la información permitirá comprender el rumor como una de las modalidades de indeterminar de la cuestión mexicana. En efecto, si la historiografía reconoce el impacto de la expedición de México en el conjunto del hemisferio occidental, las modalidades de circulación y su incidencia en los debates políticos permanecen poco estudiadas, y en consecuencia mal conocidas. Circulares diplomáticas, interpelaciones ministeriales o creación de órganos de prensa hablan de una disposición particular a sacar la cuestión mexicana de sus límites políticos inmediatos. La creación de una quincena de sociedades republicanas en el espacio de algunos meses confirma el compromiso de ver en la cuestión mexicana la suerte común al Nuevo Mundo. Situadas en las principales ciudades del espacio sur-pacifico, de Lima a Santiago, de Valparaíso a Oruro, las sociedades articulan una cartografía republicana que confirma sin ambigüedad la reactividad de la región, bien a pesar de las condiciones poco regulares de la información. Situarlas en relación al desarrollo de la cuestión mexicana autoriza a relacionarlas con la persistencia de un pensamiento que encuentra en México la posibilidad de dar al continente americano un régimen ideal para un continente gobernado durante tres siglos por una Monarquía Católica.

\section{Informaciones, escalas y formas de la información}

\subsection{De México al Sur: conexiones, tiempos y medios de circulación}

Una mirada rápida de las circulaciones hemisféricas autoriza sin ambivalencias una primera conclusión: la América hispana es, a mediados de siglo, un espacio fragmentado. Es el balance del representante mexicano a Washington, que deplora "las pocas relaciones que tenemos con ellas [las repúblicas hispanoamericanas] y de la ninguna influencia que ellas ejercen en México" ${ }^{5}$. Ahora bien, las nuevas repúblicas no han cambiado substancialmente las circulaciones que datan de la monarquía (Halperin, 2008; Irigoin, 2010). La historia diplomática de la primera mitad del siglo da fácilmente cuenta de esta fragmentación hemisférica (Núñez, 1878; De Vega, 2011)6. En los años 1820, el gobierno mexicano busca normalizar las vías de circulación con el Pacífico sur para atacarse al comercio ilegal, del cual Valparaíso era una pieza clave. Luego de algunos fracasos, le 19 abril 1843 Pedro Garmendia es nombrado Cónsul en Valparaíso. Acusado de participar en el comercio clandestino que debía atacar, Garmendia es repatriado en 1857 y su puesto

\footnotetext{
5 Matías Romero a Manuel Doblado, Washington, 4 abril 1862, n 103, Correspondencia de la legación mexicana en Washington durante la intervención extranjera. 1860-1868, v. 2, México, Imprenta del Gobierno, p. 120.

${ }^{6}$ Las líneas que siguen sintetizan esos trabajos.
} 
eliminado. Del lado chileno, el gobierno abre en 1846 un consulado en Mazatlán y otro en 1853 en San Blas, los dos en la costa pacífica. En cuanto al Perú, las cosas no son mejores; el decreto que reconoce mutuamente plenos derechos a sus ciudadanos queda en letra muerta, y sus intercambios se limitan a notificar de cambios de gobierno. Curiosamente, cuando en 1861 el gobierno peruano envía una circular alertando de los planes monárquicos de Gabriel Moreno en Ecuador, el gobierno de Juárez no responde. La rareza de los intercambios diplomáticos es aún más flagrante en Bolivia, con quien hasta 1867, México no cultiva relaciones.

La ausencia de lazos directos no implica sin embargo el aislamiento. Que se trate de individuos o escritos, la conexión entre ambos extremos tiene lugar gracias a la acción de un sinnúmero de puntos intermediarios (Lyon-Caen, 2011; Santa Cruz, 2002; Barragán et al., 2015). Cuba y Panamá son en este aspecto puntos neurálgicos de la circulación, hemisférica y atlántica (Tauzin-Castellanos, 2015). Es por vía de los corresponsales situados en estos puertos que llegan al sur las noticias de la toma de Veracruz por las tropas expedicionarias7. Los intermediarios son entonces una pieza importante en la circulación de la información, pero también de la información misma. Los circuitos y las etapas alteran la interpretación de los hechos, frente a los cual la prensa toma sus resguardos:

El Diario de la Marina [de La Havana], cuyas noticias de Veracruz solo alcanzan al 28, mientras que las que nosotros tenemos de Méjico son del 29 del mismo mes, copiando las que da un periódico de ese puerto mejicano, que se publica bajo la influencia de Almontes.

Los puertos estadounidenses tienen también un lugar asegurado en la circulación hemisférica (Bayly, 2007; Butel, 1997; Thibaud, 2017). La presencia regular de legaciones hispanoamericanas en Washington, Nueva York, California u otras ciudades costeras favorecen además el paso por el Norte de las informaciones hacia el Sur. Matías Romero, representante de Juárez en Washington, es una pieza clave en la difusión, sea organizando meetings, levantado créditos, comprando directamente armas, reclutando voluntarios o agitando la prensa (Juárez, 1972-1975; Schoonover, 1986; Taylor 1987).

La expedición abre justamente una etapa de búsqueda de canales regulares entre los dos hemisferios del continente. El Tratado de Londres por el cual

7 El Ferrocarril, 4 abril 1861; El Comercio, 18 enero 1862; El Ferrocarril, 15 mayo 1862.

8 "Vapor del Norte", El Comercio, 3 julio 1862.

9 Correspondencia de la Legación mexicana en Washington durante la intervención extranjera. 18601868, México, Imprenta del Gobierno, 1870 
Inglaterra, Francia y España se comprometen el 31 octubre 1861 a enviar fuerzas expedicionarias a México, marca una reorientación importante en las relaciones internacionales. En el Perú, el gobierno es aconsejado de abrir un consulado en Veracruz y otro en Acapulco, incitándolo a enviar la correspondencia oficial por tres vías: Saint Thomas, New York y Acapulco, "por este medio, estima El Comercio, el Perú y la América tendrán noticias frecuentes y seguras de lo que acontece en Méjico"10. El General Castilla envía en noviembre 1861 su secretario personal Manuel Nicolas Corpancho frente al gobierno de Juárez, mientras que un año después José Joaquín Pérez hace lo mismo con Ramón Sotomayor Valdés (Real de Azúa, 1978; Galdámez 1930; Brahm, 1992; Barros, 1990). Fragmentarios y raros en un primer momento, los intercambios de la costa pacifico tienden a regularizarse por medio de las misiones diplomáticas. Matías Romero se satisface de informar a sus responsables de la regularidad de la información venida por el Pacífico'11. La instalación de representantes no debe sin embargo conducir a sobreestimar el rol de la diplomacia en la calidad y fluidez de la información. La intensidad del trabajo de Romero sobrepasa en mucho al de sus homólogos hispanoamericanos, según él mismo lo reconoce:

Mientras las repúblicas hispanoamericanas estén representadas como en la actualidad, no hay esperanza de adelantar nada. De los cinco representantes que dichas repúblicas tienen ahora en Washington, incluyendo a México, tres pertenecen al partido extremista conservador de la América del Sur, y solo el ministro del Perú y yo formamos la facción liberal, si es que puede llamarse liberal al señor Barreda12.

La apertura hacia el Este compromete también las capitales europeas y, por extensión, el Rio de la Plata. La necesidad de informaciones fiables y de conocer el estado de avance de la expedición abre la posibilidad de los hispanoamericanos residentes en Europa de constituirse en motores de esta red política en gestación (Guerra, 1989; Sanhueza, 2007; Gutiérrez, 2009; Delli Quadri, 2014). Desde El Havre, el cónsul peruano Luís Benjamín Cisneros acompaña su correspondencia de extractos de prensa y de copias de documentos oficiales. Sus escritos encuentran un

\footnotetext{
10 “Crónica de la Capital”, El Comercio, 8 julio 1862.

11 Matías Romero al Ministro de Relaciones Exteriores, Washington, 20 octubre 1862, Correspondencia de la Legación mexicana en Washington durante la intervención extranjera. 1860-1868, v2, 1862, Imprenta del Gobierno, 1870, p. 545.

12 Matías Romero al Ministro de Relaciones Exteriores, Washington, 29 noviembre 1862, Correspondencia ... op. cit., p. 640.
} 
público más amplio cuando reemplaza a José María Samper en la correspondencia francesa de El Comercio. La prensa hispanoamericana en Europa, como El Correo de Ultramar o El Eco Hispano-Americano tienen también una difusión asegurada en el hemisferio (Olguín, 1984). Incluso al precio de idas y vueltas que no hacen sino anunciar lo que la opinión ya conoce en detalle. Es el ejemplo del Ferrocarril de Santiago, que informa en un mismo número del retiro de las tropas británicas y españolas de Veracruz, y de la victoria de las fuerzas de Juárez en Puebla1 ${ }^{3}$.

En cuanto a la circulación en el Sur, la importancia de las ciudades costeras confirma sin reticencias la importancia de los circuitos marítimos. El Callao, Lima, Copiapó, La Serena Valparaíso y Santiago están vinculados a Nueva York, Veracruz, Acapulco, La Havana, Panamá o Rio de la Plata por una red que sigue en lo más importante el trazado del periodo colonial (Demélas, 1992; Villalobos, 2002 ; Parodi y Miranda, 2014; López Beltrán, 2016). Las innovaciones técnicas como el vapor, el tren o el telégrafo revolucionan la frecuencia de la prensa, como lo muestra el trabajo de las imprentas luego de la llegada del Vapor, pero no alteran substancialmente los circuitos.

Si los circuitos marítimos juegan un rol preponderante en la circulación atlántica, esta adopta formas diversas cuando se trata de conectar los valles del interior andino (Osterhammel, 2015). Allí, el vapor cede en el mejor de los casos al cable telegráfico. Es por esta vía que Santiago y Lima siguen en tiempo real las informaciones que llegan a Valparaíso y al Callao. En la mayoría de los casos, estas viajan a lomo de mula, caballo, o llama, o gracias a los numerosos hombres que conectan a pie un valle con otro (Demélas, 1992; López Beltrán, 2016; Walker, 2001). Tres grandes ejes favorecen esos intercambios. El primero y más importante a mediados de siglo vincula Islay, Arica y Tacna con La Paz, cuyo comercio crece considerablemente a lo largo del siglo. El ascenso de La Paz vincula el comercio de los valles andinos a los circuitos de la costa pacífica también pasando por Puno y Arequipa (Rosemblitt, 2018; Condori, 2010). El segundo, conocido como la Ruta de la Plata relaciona el eje Potosí-Sucre con el Sur boliviano por Arica. El tercero, conocido como la Ruta del Pescado vincula Potosí y Cobija, pasando por Calama (Demélas, 1992; López Beltrán, 2016).

Si se observa el tiempo de circulación, es posible constatar que tres meses necesitan para conectar México DF y La Paz ${ }^{14}$. El viaje hacia los valles del interior es sin duda el más sinuoso y representa una parte importante del tiempo de circulación. Entre el Callao y La Paz la comunicación puede tomar hasta cinco

\footnotetext{
13 La Voz de Chile, 25 julio 1862.

14 "Méjico", El Telégrafo, 23 marzo 1862.
} 
semanas si las condiciones son favorables15. Arequipa, Ayacucho, La Paz, Sucre o Potosí se incorporan así a esta cartografía múltiple, hecha de innovaciones técnicas y formas tradicionales.

La dependencia del interior respecto a las ciudades de la costa sitúa a estas últimas en primera línea. El interés del Pacífico-Sur por el desarrollo del conflicto en México no puede encontrar satisfacción sino a través de estos canales de circulación. Por el vapor, el telégrafo o aun por fuerza animal o humana, las circulaciones toman a veces desviaciones inusitadas, en todo caso raramente directos, entre une hemisferio y otro. Es gracias al rol de los intermediarios que esta cartografía revela su fisonomía, permitiendo a los escritos y a los hombres hacer la experiencia de un mundo interconectado, aunque sea por vías diferidas.

\subsection{La indeterminación y el rumor: dos formas complementarias de la información}

Un aspecto importante de la cuestión mexicana es la dificultad existente en el Pacífico-Sur para obtener informaciones fiables, lo que impide la apreciación de su justo valor. "Los asuntos de Méjico no tienen todavía un carácter definitivo"16; a más de un año de comenzado el conflicto, los observadores deploran aun la poca claridad de las informaciones disponibles. Indeterminación estructurante, que no hace sino develar en los escritos la lucha implícita por controlar el significado de las cosas, dando origen a un sinnúmero de rumores (Aldrin, 2005). Los rumores borran el origen de la información, y abren el hecho a toda suerte de posibilidades. Incluso en círculos reacios a este tipo de imprecisiones. Desde Washington, Francisco Solano Astaburuaga informa al Gabinete de Santiago los rumores que se transmiten en el Norte:

La Inglaterra y la Francia más parecen que aprestan la anunciada expedición para el envió de respetables fuerzas navales al golfo mejicano que estén a la mira de una oportunidad en que convenga obrar en favor de los Confederados 17 .

\footnotetext{
15 "Méjico", El Comercio, 29 enero 1862; El Telégrafo, 10 marzo 1862.

16 Juan Antonio Ribeyro a Federico Barreda, Lima, a Washington, 19 junio 1863. Archivo Ministerio de Relaciones Exteriores Lima, CC51 - Copiador de la Correspondencia dirigida a las Legaciones del Perú en América

17 Francisco Solano Astaburuaga al Ministro de Relaciones Exteriores, Washington, 20 noviembre 1861. Archivo Nacional de Santiago, fondo MRE, v. 85, n 19.
} 
El rumor encuentra en la prensa su terreno más fértil. Un folleto ampliamente difundido en Lima, Santiago y Valparaíso atribuye a la intervención el proyecto de dividir México18. En Valparaíso, El Tiempo ofrece otro caso concreto. Su edición del 25 abril 1862, alerta sobre un eventual acuerdo entre el General Urquiza y Napoleón III para restituir la monarquía en el Rio de la Plata:

El apetito se aumenta comiendo: se habla de proyectos más vastos y que comprenden otras repúblicas de la América del Sur. Así se desea saber si se trata de la cuestión de crear un nuevo reino que comprenda las repúblicas de Venezuela, del Ecuador, y de la Nueva Granada, en que tendría por capital a Caracas; y en seguida de un nuevo reino, que comprendería los Estados del Plata teniendo por capital a Buenos Aires. Hasta se agrega que el general Urquiza, que opina por este proyecto, es esperado por Francia, a donde vendrá a entenderse con el emperador Napoleón 19 .

Lo que perturba del rumor no es la imprecisión sino su función de tantear la opinión. Eso le da una fuerza política difícil de hacer enfrentar por los medios formales. Es la dificultad que reconoce la prensa semioficial en Bolivia que se resigna a informar a sus lectores que, "las noticias que se tienen de Méjico son contradictorias, asegurándose por unos que las últimas ventajas pertenecen a los mejicanos y por otros al ejército francés" 20 . La desatención por el origen aumenta la masa de rumores en circulación. Prensa, cartas, folletos, extractos, hojas sueltas; los impresos vehiculan informaciones sobre todo comentadas, sometiendo los hechos al buen parecer de los comentadores. La dificultad de separar lo real de lo ficticio y el hecho del comentario da a la cuestión mexicana una importancia inédita en el Pacífico-Sur. La dificultad de despejar la nube de rumores incide directamente en la política de los gobiernos. A un año y medio de organizada la expedición, el presidente José Joaquín Pérez confía al representante de Madrid en la capital, Santiago Tavira, su decisión de no protestar, fundándose en las noticias poco confiables al respecto21. En su mensaje anual al Parlamento, el General Castilla no ignora el carácter dudoso de las informaciones disponibles, aun las de su entorno más cercano: "se hace difícil creer que tales rumores [la monarquización de México]

\footnotetext{
18 Antinóo, Reconquista de las colonias Sur-Americanas, Lima, Imprenta de José S. Macias, 1862.

19 El Tiempo, 25 abril 1862

20 La Voz de Bolivia, 5 noviembre 1862.

21 Santiago Tavira al Primer Secretario de Estado, Santiago, 17 agosto 1861, Archivo Histórico Nacional de Madrid, fondo MAE, H1438, doc. $\mathrm{n}^{\circ} 85$.
} 
sean fundados, o que tal pensamiento si ha existido, prevalezca, o que pueda realizarse"22.

El suspenso de las fuentes atraviesa de un punto a otro el Pacífico-Sur y es, al decir verdad, un aspecto que no preocupa a los editorialistas. "Uno de nuestros amigos [...] nos ha remitido" 23 , "dícese que" o aun "una carta [...] dice que" son tantas fórmulas que satisfacen, cierto, una demanda en aumento, pero que diluyen la autoridad de la información ${ }^{24}$. Efecto de retórica o simulación de indecisión, la prensa actúa libremente en el intersticio que separa los hechos de los lectores ${ }^{25}$. Al punto de poder utilizar la cuestión de México para otros fines, como lo muestra el anuncio de la venta de un piano bajo el título "Viva Méjico"26.

La disolución del origen da a los editores de prensa un rol decisivo en la manera de trabajar la información. En las ciudades costeras como en el centro de los Andes, los rumores hacen pensar en el interés particular de España en la aventura mexicana27. Frente a esos ruidos, El Mercurio de Valparaíso, propietario del español Santos Tornero, lanza una campaña de desmitificación, comenzando por denunciar el mal ánimo de la prensa en el Perú:

Grande es el empeño con que ciertos escritores del Perú se esfuerzan, de un tiempo a esta parte, en hacer creer que el gabinete español tiene intención de reconquistar a sus antiguas colonias, y que su proyecto es segundado, ya por algunos gobernantes de las repúblicas hispano-americanas, ya por los hijos de la Península, que residen en este continente28.

Lo que es afirmado es rápidamente desmentido, y el impreso, en sus usos múltiples, se adapta convenientemente a esas fluctuaciones. Es el caso de una hoja suelta que circula en Tacna, que recurre a un vocabulario figurativo para amenazar a los resientes europeos, acusados por defecto de complicidad con los planes imperiales en México: "hay muy fundadas esperanzas de que vengan pronto órdenes del Supremo Gobierno para que se haga de todos los extranjeros un inmenso picante

22 Mensage que el Libertador Presidente de la República dirige a la Legislatura de 1862, Lima, Imprenta del Gobierno, 1862, p. 5. Ver también, Memoria que presenta el Ministro de Estado en el departamento de relaciones Exteriores y Culto al Congreso Nacional de 1862, Lima, Imprenta de la Época, 1862.

23 El Telégrafo, 23 marzo 1862.

24 El Ferrocarril, 23 enero 1861.

${ }_{25}$ La Voz de Bolivia, 5 noviembre 1862.

26 El Ferrocarril, $1^{\text {ro }}$ junio 1862.

27 Francisco Solano Astaburuaga al Ministro de Relaciones Exteriores, Washington, 10 noviembre 1861. Archivo Nacional de Chile, fondo MRE, v. 85, doc. n¹8; El Telégrafo, 18 diciembre 1861.

28 "La España y las Repúblicas Americanas", El Mercurio, 29 noviembre 1861; El Telégrafo, 21 diciembre 1861. 
con ají colorado"29. El documento, que llegó hasta el gabinete de Isabel II, originó la coordinación estrecha entre las legaciones europeas para asegurarles la vida y los bienes, así como también para alertar al gobierno de las amenazas ${ }^{3}$.

En las redacciones y gabinetes, pero también en los salones, calles y plazas, los rumores se expresan sin impedimentos. Esos espacios de oralidad terminarán marcando con su sello la producción de la palabra escrita (Uribe, 1974; Kalifa et al., 2001; Walker, 2001; Demélas, 1992). Con esto, la opinión exhibe la persistencia de formas que recuerdan el régimen de publicidad bajo la Monarquía hispánica (Lempérière, 2004). Los recursos morales juegan un rol decisivo en esta gramática de la información. La prensa, en su trabajo de hacer público los asuntos de la cité, es pensada como un indicio de salud de la comunidad política. En su exaltación republicana, esta certifica la ejemplaridad moral y la honorabilidad de los ciudadanos, vecinos y hombres políticos. La denuncia, la acusación, la polémica operan como medios de hacer visible la desviación moral en el seno de la comunidad. Por otra parte, el vicio, la tentación y la traición expresan los males de un cuerpo social y político en disfunción. Es en estos términos que El Tiempo denuncia, la ceguera de la opinión sobre las reales intenciones de los agitadores ${ }^{31}$. Por su parte, El Ferrocarril replica, criticando la intención del Tiempo de "seducir maliciosamente al público"32. De un impreso al otro, las formas de esta cultura comunitaria se dejan ver en el conjunto de referencias cruzadas que se adaptan globalmente a las exigencias de los usos más formales de la palabra impresa. Es a través de los rumores que la cuestión mexicana adquiere en los debates del PacíficoSur su primera fisonomía. Es también gracias a ella región evalúa las implicaciones locales de la aventura mexicana.

\section{El Pacífico Sur, ¿un terreno sensible a la monarquía?}

\subsection{La difícil caza del pensamiento monárquico}

El republicanismo sur-pacífico no se puede pensar al margen de la persistencia de un pensamiento nostálgico de la Monarquía en América. La historiografía ha tradicionalmente minimizado el impacto del pensamiento monárquico en América hispánica, y los estudios que lo vinculan a las tensiones

\footnotetext{
29 Antropófagos, Satisfacción a los manes del General Iguain, Tacna, Tipografía del Provenir, 7 enero 1862.

30 José Merino Ballesteros al Primer Secretario de Estado, Lima, 26 enero 1862. Archivo Histórico Nacional de Madrid, Fondo MAE, Lima (1856-1930), H1931, doc. $n^{\circ} 2$.

31 El Tiempo, 6 marzo 1862.

32 El Ferrocarril, 19 abril 1862.
} 
atlánticas de mediados de siglo son contados (Pani, 2001; 2002). Paradojal que pueda parecer, las expresiones monárquicas no se reducen a indicios aislados, y el naciente movimiento republicano así lo entiende. Frente al peligro que cae sobre México, la campaña republicana consiste en denunciar públicamente los monarquistas locales. La cuestión de México se presta así para arreglar viejas querellas políticas en la región. El desarrollo de esta campaña muestra hasta qué punto la región entiende su destino vinculado al desenlace del conflicto en el Norte. El limeño Ricardo Palma revela bien las características de esta campaña. Exiliado en Valparaíso luego del atentado fallido contra el presidente Castilla, Palma sigue las tensiones atlánticas en compañía de otros exiliados. La campaña estalla cuando El Mercurio le rechaza un virulento artículo contra la anexión de Santo-Domingo a la corona de España. Frente al rechazo de su propietario, el español Santos Tornero, Palma decide su publicación en hoja suelta, vinculándolo con los planes monárquicos de España en el Nuevo Mundo: "Por los visto, nuestra antigua metrópoli [...], cuenta en Chile con colaboradores activos para su obra de próxima reconquista" 33 . La acusación sin dudas sorprendió a la prensa, incluso a los sectores liberales. Tanto más cuanto Tornero es en esos años tenido por benefactor de la prensa liberal y moderada (Lastarria, 1878: 388). La denuncia de la reconquista hispánica y los ataques a Tornero, ponen a la defensiva al representante español en Chile. La investigación conduce a Santiago Tavira a identificar la hoja con el exilio en Valparaíso, particularmente "los emigrados peruanos General Rivas, Francisco Javier Mariátegui y otros varios me citan también al Cónsul peruano en Valparaíso" 34. Algunos días más tarde, se publican las descargas del editor, acusando directamente al presidente peruano de sembrar las discordias para aprovecharlas a su favor 35 . El 12 de diciembre 1861 El Chalaco decide de entrar en el debate y denuncia en estos términos la complicidad del Mercurio:

el Sr. Tornero, desde que ha negado las columnas de su periódico para la inserción de un artículo contrario a la reconquista, ha manifestado tácitamente que esta por ella; y el

\footnotetext{
33 Dos Republicanos: Pablo et Job, Valparaíso, 26 noviembre 1861, Imprenta Del Universo de G. Heleann. Publicado también en Cruz, 1933: 118-120.

${ }_{34}$ Santiago Tavira al Primer Secretario de Estado, Santiago, 2 diciembre 1861. Archivo Histótico de Madrid, fondo MAE, H1438, doc. ${ }^{\circ} 84$. Santos Tornero, afirmará años después que se trataba de exiliados ecuatorianos. Santos Tornero, Reminiscencias de un viejo editor, Valparaíso, Imprenta del Mercurio, 1889.

35 "La España y las Repúblicas Americanas", El Mercurio, 29 noviembre 1861, publicadas también en El Telégrafo, 21 diciembre 1861. Ver también Santos Tornero, Reminiscencias de un viejo editor, Valparaíso, Imprenta del Mercurio, 1889.
} 
pueblo chileno debe desde luego considerarle como enemigo suyo ${ }^{36}$.

Otros artículos se suman también a la discusión, desplazando el terreno de un asunto personal a una cuestión propiamente política. En La reconquista y los defensores de la independencia americana, Florentino González acusa a la prensa local de estar pagada por el gobierno peruano37. La acusación es recibida como una verdadera provocación, por la prensa peruana como por los mismos exiliados de Castilla en Valparaíso, que no tardan en replicar afirmando su independencia e incluso saludando la política activa del Perú en materia de relaciones internacionales ${ }^{38}$. El articulo inicial de Palma conduce así a Florentino González. Colaborador del Mercurio y editor del Tiempo de Valparaíso, González tiene en efecto de qué despertar las sospechas republicanas. Hombre político neogranadino reconocido, su proposición de anexar la Nueva Granada a los Estados-Unidos lo aleja de sus antiguos camaradas liberales. En el Perú y luego en Chile, González se atrae la animosidad republican a causa de su colaboración con el presidente Gabriel García Moreno (1861-1865) y el General Juan José Flores, conocidos monarquistas exaltados (Figueroa, 1900: 96; Cardona, 2014; Tamayo, 2017). Su afinidad con la causa imperial mexicana puede leerse en las páginas del Tiempo, donde un artículo sobre él y muy probablemente redactado por él mismo, expresa:

En cuanto a Méjico, menos le disgustaría a González una monarquía constitucional que proscribiese la esclavitud, que el verlo conquistado poco a poco por los confederados del Sur y planteada en él la Republica de la Carolina, de la Georgia y del Alabama, con la bellísima institución de la esclavitud 39 .

Por su parte, Gabriel García Moreno es otro objetivo directo de la crítica republicana. Queriendo hacer frente a las tensiones con el gobierno de Castilla, García Moreno conduce una campaña para comprometer el Segundo Imperio en la creación de un protectorado capaz de reagrupar Ecuador y Perú (Van Aken, 1989; Buriano, 2015; Henderson, 2008). Para ello envía Antonio Flores, hijo del General Juan José Flores, en calidad de representante de Quito en Londres y París. En la carta que dirige el 20 de enero de 1863 a Napoleón III, Flores sitúa el Ecuador del lado de la política imperial en el Nuevo Mundo: "Nada sabría contribuir más eficazmente a la

\footnotetext{
36 El Chalaco, 12 diciembre 1861.

37 F. G., "La reconquista y los defensores de la independencia americana", El Mercurio, 9 enero 1862.

38 Ricardo Palma, "Revista de la Quincena”, Revista de Sud-América, 10 enero 1862.

з 9 "Exactitud y mala fe", El Tiempo 6 marzo 1862.
} 
regeneración de esas jóvenes república que la instalación de un poder estable y sólido en México"40. Para Castilla, como para tantos otros, se trata de una provocación explícita, lo que permite de comprender mejor su apoyo a las tentativas por derrocarlo (Van Aken, 1989: 261).

Las formas en que la cuestión mexicana se implica en los debates políticos del Sur no derivan únicamente de querellas entre caudillos. Paradojal que pueda parecer, una explicación más profunda implica integrar la parte del pensamiento monárquico en la construcción de las nuevas republicas hispanoamericanas. En efecto, la idea monárquica juega un rol importante en la política hispanoamericana, incluso a mediados de siglo. En 1858 son publicadas en París las memorias póstumas del Mariscal Riva-Agüero. Veterano de las guerras de Independencia, estas presentan una justificación de las tentativas que buscaban en los años 1820 dar al Perú un príncipe español, proyecto respaldado en su momento por otros héroes de la independencia. Haciendo un balance de las primeras décadas republicanas, Riva-Agüero radicaliza su pesimismo, ya conocido gracias a sus escritos editados en esos mismos años en Londres y Santiago (Riva-Agüero, 1824; 1828). Sus Memorias y documentos para la historia de la independencia del Perú y causas del mal éxito que ha tenido esta, defiende una solución que sorprende por su similitud con los sucesos de México: "La intervención europea sería recibida con entusiasmo por los peruanos, que mirarían en ella su libertad y su prosperidad futura" (Riva-Agüero, 1858: 8) El caso de RivaAgüero no es único en el Perú y debemos también a otros la persistencia de un pensamiento crítico de los fundamentos republicanos ${ }^{4}$.

Incluso del otro lado del océano, el pensamiento monárquico vuelve a la América hispánica por vía de la recuperación de los derechos de Isabel II sobre las nuevas republicas. Aquí también, es posible documentar una serie de intereses que conducen a pensar que el pensamiento monárquico goza, incluso a mediados de siglo, de buena salud. En Europa, las intenciones de un retorno a la monarquía en el Nuevo Mundo se expresan teniendo en cuenta el inventario de sus riquezas. En este punto, las islas Chincha pueden bien servir de ejemplo. Fundamental en la economía de país, las islas formadas de guano favorecen un desarrollo sin precedentes en el Perú. Entre 1840 y 1880, once millones de toneladas son exportadas, principalmente a Europa; si entre 1846-1847 representa el 5\% de las exportaciones, en 1868-1875 es un $80 \%$ (Bonilla, 1984; Gootemberg, 1989; Portocarrero, 2009). Los observadores europeos los saben bien. En marzo 1859 el fotógrafo y viajero Louis de Clercq aconseja las autoridades del Segundo Imperio retener las islas en respuesta a la

40 Antonio Flores a Napoleón III, París, 20 enero 1863. Centro de Archivos Diplomáticos de Francia, sede La Courneuve. Fondo Affaires diverses Politiques, Ecuador, 26ADP1, f ${ }^{\circ} .89$.

41 Un Thaboriano, Exámen comparativo de la Monarquía y de la República, Lima, 1867. 
indiferencia del gobierno de Lima a dar satisfacción a las reclamaciones Durhin (Novak, 2005)42. El consejo se funda en numerosos estudios químicos que desde fines del siglo XVIII avalan los beneficios del guano en la explotación agrícola43. El interés económico se observa también del otro lado de los Pirineos. En España, un sector de la prensa interpela la Corona sobre los asuntos pendientes en el PacificoSur. En junio 1861 La España de Madrid publica una serie de artículos que respaldan el derecho de Isabel II a hacer efectiva su soberanía sobre las islas. El argumento se funda en el no reconocimiento de la independencia del Perú, y en consecuencia, en el derecho de la Corona a tomar posesión de, "unos peñascos que contienen infinitamente más riqueza que muchas comarcas adelantadas de Europa"44. El texto, ampliamente difundido por la prensa de diversos colores políticos, prueba que la idea goza de una recepción relativamente favorable45. En Santiago, El Ferrocarril publica un extracto del Español de Ambos Mundos, que muestra bien la disposición de un sector político de la península: "los grupos de Chincha [...] pertenecen a España lo mismo que le pertenece el Perú"46. Si España, prosigue el artículo, reconoció la independencia de la tierra firme, no sucede lo mismo con los dominios insulares. Por ello, declara, "podrá recobrar [...] la isla de San Lorenzo, que está en la embocadura del Callao, i otra multitud de islotes desde California al cabo de Hornos" 4 . Pero no es únicamente en la prensa de Madrid que la atracción por las islas es observable. En 1863 el vicecónsul español en Valparaíso envía al gabinete de Madrid informes químicos sobre el guano de Chincha, acompañados de los trabajos de Ignacio Domeyko, entonces profesor de minas en la Universidad de Chile ${ }^{48}$. Es justamente reconociendo los riesgos de una alianza entre monarquistas de ambos lados del Atlántico que el movimiento republicano se articula en la costa sur-pacífico. La

\footnotetext{
42 Archivos Diplomáticos de Francia, sede La Courneuve. Fondo Affaires Diverses Politiques, 54ADP3, Pérou, 1814-1896. Sobre la reclamación Durhin, ver Question entre la France et le Pérou par suite de l'Emprisonnement du Sujet François Paul Durhin, Lima, Imprenta de Aurelio Alfaro, 1862. Un ejemplar se encuentra en el Instituto Riva-Agüero.

43 Guyton, Monge, Berthollet, Fourcroy, Adet, Hassenfratz, Seguin, Vauquelin, C. A. Prieux, Chaptal, Parmentier, Deyeux Bouillon-Lagrange y Collet-Descostils, Annales de Chimie, ou recueil de mémoires concernant la chimie et les arts qui en dépendent, et spécialement la pharmacie, v. 56, París, Librairie Bernard, 1805 ; J.-C. Delamétherie, "Analyse du Guano", Journal de Physique, de Chimie, d'histoire naturelle et des arts, avec des planches en taille-douce. v. LXII, París, Imprimerie et Librairie Courcier, enero 1806. Finalmente, "Guano du Pérou et des autres parties de l'Amérique", Annales de la Société universelle pour l'encouragement des arts et de l'industrie, julio 1855, año 5, nº 7, pp. 103-109.

44 “Cuestión importante", La España, $1^{\text {ro }}$ junio 1861, reproducido en La América, 24 junio 1861. Ver también La España, 12 y 18 junio 1861.

45 La Epoca, $1^{\text {ro }}$ et 12 junio 1861 ; La América, 24 junio 1861.

46 El Ferrocarril, 7 agosto 1861, copiado del Español de Ambos Mundos.

47 Ibid.

48 Antonio Agacio al Primer Secretario de Estado, Santiago, $1^{\text {ro }}$ agosto 1863. Archivo Histórico de Madrid, fondo MAE, H2090. Ver también carta del 9 septiembre 1863.
} 
cuestión mexicana reabre en el Sur el viejo debate sobre el régimen político. Estos casos revelan hasta qué punto la defensa republicana actúa frente a un pensamiento que busca todavía el régimen apropiado para territorios gobernados durante tres siglos por una monarquía católica.

\subsection{La estabilización de la cuestión mexicana y el nacimiento de la sociabilidad republicana}

El pensamiento monárquico se ve favorecido por la mala imagen que en un primer momento las repúblicas del Sur tienen de México. Esta primera imagen conocerá transformaciones importantes destinadas a instalar en el debate la imperiosa necesidad de defender la república en el Nuevo Mundo. Así, en el transcurso de algunos meses, de ser considerado un país de mala reputación, el hemisferio comienza a pensar México como el más reciente gran momento histórico del hemisferio (Caillet-Bois, 1963; López, 1969; Covo, 1971; Salomon, 1975; Meyer, 2014). El desplazamiento esta ritmado por el curso de los hechos en el Norte, pero también por la uniformización de la opinión en el Sur. La intervención aliada, cuyo primer objetivo era el reclamo de las deudas internacionales, se transforma al cabo de algunos meses en la expedición francesa en México. Es al término de estas transformaciones y gracias a ellas que ven la luz en las principales ciudades de la región las sociedades republicanas.

La imagen de México en los años 1850 está fuertemente ligada a la derrota de la guerra contra los Estados-Unidos, terminada en 1848 (Zoraida y Meyer, 2013; Hale, 1957). En su diario personal, publicado en Santiago en 1856, Benjamín Vicuña Mackenna la evoca en estos términos: "los mejicanos han sido más desgraciados que cobardes, el pueblo se condujo con patriotismo y abnegación, pero los la cobardía y la rivalidad de sus jefes lo perdieron y humillaron"49. A la derrota, se suman los interminables conflictos fratricidas. El Ferrocarril de Santiago habla a inicios de 1861 de la "malhadada república"50, o aun de la anarquía provocada por la guerra civil, "que enervará completamente las fuerzas del país si no tratan de cortarla por medio de una intervención las potencias europeas"51.

Hacia fines de 1861 la prensa imperial encuentra en las columnas de la región un espacio asegurado para explicar las intenciones de la expedición52. Los

\footnotetext{
49 Benjamín Vicuña Mackenna, Pájinas de mi diario durante tres años de viajes 1853-1854-1855, Santiago, Imprenta del Ferrocarril, 1856, p. 25.

50 "Dos partidos en México", El Ferrocarril, 13 febrero 1861.

51 El Ferrocarril, 30 marzo 1861.

52 Sobre los argumentos del Segundo Imperio, ver Michel Chevalier, Lettres sur l'Amérique du Nord, París, 1844. También, Lecaillon, 1994; Avenel, 1996; Milza, 2004.
} 
argumentos imperiales no modifican en nada la percepción negativa que gravita sobre México, pero permite hacer leer la compatibilidad entre los dos. Lo confirma implícitamente El Ferrocarril, periódico que al cabo de unos meses será paradojalmente uno de los órganos de la causa republicana. Su edición del 30 de octubre reflexiona en estos términos sobre las implicaciones de la intervención, "Ojala que la amenaza extranjera sea un elemento de unión para los mejicanos, aunque bien poco puede esperarse del patriotismo i enerjia de un pueblo que se dejó dominar por un puñado de yankees" 53 . Una semana más tarde, retoma la cuestión mexicana a la luz del pesimismo: "la expedición armada con que [...] amenazan a esa república, ha despertado en las desorganizadas hordas que la despedazan [...] la rabia salvaje propia de la barbarie i de la cobardía" 54 . Por su parte, comentando la prensa internacional, El Comercio ve en la expedición un freno al avance de los Estados-Unidos hacia el Sur: "el momento ha llegado de que la Europa les ponga una valla [a los Estados Unidos] para impedir que la raza latina sucumba más tarde en este Continente" 55.

Un nuevo episodio altera este primer estado de cosas y logra el desacuerdo entre el pesimismo sobre México y los argumentos imperiales. El 19 de febrero 1862 los representantes de Juárez y de la expedición firman los acuerdos de La Soledad. El documento tiene un rol mayor en el desarrollo del conflicto. En primer lugar, despeja los rumores de favorecer un cambio de régimen político en el país, pues reconoce implícitamente el gobierno de Juárez como interlocutor. A cambio, Juárez autoriza el desplazamiento de la tropa hacia el interior de Veracruz, con el fin de evitar las enfermedades de la estación. En segundo lugar, de romperlos, las fuerzas aliadas se comprometen a volver a Veracruz. La ruptura en abril 1862 se traduce en el reembarco inmediato de las tropas españolas y británicas. La mantención de las fuerzas francesas contribuye a develar, de una vez por todas, las verdaderas intenciones de lo que uno de sus propagandistas definió como la plus grande pensée du règne (Lecaillon, 1994; Avenel, 1996; Milza, 2004; Gouttman, 2008). La llegada de refuerzos de París con el General Miramón, adversario de Juárez durante la Guerra de Reforma (1858-1861), no hacen sino confirmar la colaboración entre la oposición a Juárez y los agentes del Segundo Imperio5 6.

La llegada de las fuerzas expedicionarias representa un cambio importante en los debates locales sobre la cuestión. Entre marzo y abril el conflicto armado se impone como una realidad cuyas implicaciones quedan aún por desvelar. Es el

53 El Ferrocarril, 30 octubre 1861.

54 El Ferrocarril, 6 noviembre 1861 (corresponsal de Lima, en carta fechada del 26 octubre 1861).

55 El Comercio, citado del New York Times, 31 enero 1861.

56 El Ferrocarril, 23 enero 1861. 
momento en que el Pacífico-Sur abandona e pesimismo sobre México y reflexiona sobre las implicaciones concretas para el Nuevo Mundo. Redactor en jefe del Mercurio de Lima, Manuel Anastacio Fuentes es una de las raras figuras que polemizan con la virulencia del naciente movimiento republicano. Eso no le impide reconocer lo esencial de la inquietud: "la conducta de la España, de la Francia y de la Inglaterra, no puede dejar de provocar las susceptibilidades y las desconfianzas de todas las Repúblicas Hispano-Americanas" 57 . Le sentimiento que con México es el Nuevo Mundo que se revela a las ambiciones europeas se extiende en la opinión. Se abre así un segundo momento, marcado por las sospechas concretas de un cambio de régimen en México, cuyas implicaciones para el Nuevo Mundo no son desconocidas. Un folleto anónimo publicado en París advierte sobre la sobre posibilidad de alterar el régimen político: "en la sociedad entera los intereses creados, las ideas acreditadas y el orden establecidos están tan en armonía con las instituciones, que ningún partido osa atacar la organización republicana"58. Otro texto denuncia la intervención en México como "un atroz insulto a los pueblos Sudamericanos, y de hecho una amenaza directa a sus intereses legítimos" ${ }^{59}$. En el Perú, la prensa se conduce hacia conclusiones radicales:

No deja duda ya de que la Francia asume en Mejico un papel incompatible con la autonomía de esa República y revela al mismo tiempo el plan interesado que el gabinete de las Tullerias ha llevado a cabo en la triple alianza contra Mejico. Hoy, pues, es un hecho que el propósito de la Francia de intervenir en la organización interior de la república mejicana6 0 .

La Soledad transforma la fisonomía de la expedición, en favor de la defensa de México y de la república en el Nuevo Mundo. El conflicto, vociferado por los más alarmistas como una guerra por modificar el régimen político, renueva así la corriente de críticas antiimperiales desarrolladas desde los años 1850 en ocasión de los conflictos con los Estados Unidos (Gobat, 2013, Mignolo, 2005). El cambio de percepción se acompaña de una colaboración más estrecha entre la prensa critica de la expedición. En este movimiento, El Siglo XIX y El Monitor Republicano, órganos de

\footnotetext{
${ }_{77}$ Manuel Anastacio Fuentes, Méjico y la alianza Hispano-Anglo-Francesa, Lima, tipografía de la época por L.E. del Campo, 1862, p. 16

58 El Perú y la influencia Europa. Paris, Librería Universal, 1862, p. 19.

59 Sebastián Jiménez, Las repúblicas americanas y el imperio francés, Lima, Imprenta del Comercio, 1863, p. 7.

60 La América, 24 mayo 1862.
} 
las fuerzas mexicanas que luchan contra el imperio, adquieren un espacio privilegiado en la prensa antiimperial del Pacífico-Sur. Una nueva consigna política reagrupa detrás de sí los republicanos más decididos. Guillermo Matta resume en estos términos las mutaciones de la prensa: “desengañémonos, defender a Méjico es defendernos a nosotros mismos" 61 . La percepción según la cual la suerte de México anuncia el avenir de las demás repúblicas hispanoamericanas logra instalarse con éxito en el debate regional. La acompaña la creación de una decena de sociedades republicanas que se dan por tarea la defensa de la República en el Nuevo Mundo.

Las reacciones sud-pacíficas son el resultado de un doble movimiento. Por un lado la indeterminación de la cuestión mexicana y la amplitud de las circulaciones obligan a tomar precaución de los rumores que circulan en torno a la expedición. Por otro, la expedición vuelve a abrir el debate sobre el régimen político ideal para el Nuevo Mundo. Las acusaciones de colaboración con los planes de reconquista revelan hasta qué punto es temida una eventual alianza de monarquistas en el Nuevo Mundo. Frente a la amenaza, las reacciones que conoce la región recuerdan que la idea monárquica está lejos de haber desaparecido de los debates políticos. En tal sentido, la cuestión de México revela los trazos de una profunda historia común.

\section{Bibliografía}

Aldrin, P. (2005) : Sociologie politique des rumeurs. París, Presses Universitaires de France.

Avenel, J. (1999): La campagne du Mexique (1862-1867) la fin de l'hégémonie européenne en Amérique du Nord. París, Economica.

Barragán, R., Lema, A. y Mendieta, P., coords. (2015): Bolivia y su historia. Los primeros cien años de la Répública 1825-1925. La Paz, Coordinadora de Historia.

Barros Van Buren, M. (1990): Historia diplomática de Chile (1541-1938). Santiago, Editorial Andrés Bello.

Basadre, J. (1946): Historia de la República del Perú, v. 1, 1822-1866. Lima, Cultura Antártica S. A.

Basadre, J. (2002): La iniciación de la República: contribución al estudio de la República, v. 1. Lima, Fondo Editorial Universidad Nacional Mayor de San Marcos.

61 Guillermo Matta, "La invasión de Méjico", La Voz de Chile, 30 marzo 1862. 
Bayly, C. (2007) : La naissance du monde moderne (1780-1914). París, Les éditions de l'atelier/Le Monde.

Bonilla, H. (1984): Guano y burguesía en el Perú. Lima, IEP.

Borie, C., Castro, V., Varela, V. y Aldunate, C. (2016): “Cobija y sus vías de comunicación con el interior de Atacama. Desde la colonia hasta la guerra del salitre", Diálogo Andino, (49), pp. 209-226.

Brahm, E. (1992): "La crisis del conservantismo chileno en la segunda mitad del siglo XIX. Política, gobierno y régimen de gobierno en el itinerario intelectual de don Ramón Sotomayor Valdés", Revista Chilena de Derecho, 19(1), pp. 7-33.

Buriano, A. (2015): "Entre el protectorado y la República del Sagrado Corazón: el Ecuador garciano, 1860-1875”, Historia Mexicana, 65(2), pp. 561-597.

Butel, P. (1997): Histoire de l'Atlantique de l'Antiquité à nos jours, París, Perrin.

Caillet-Bois, R. (1963): “Argentina y la intervención europea en México en 1862", Historia Mexicana, 12(4), pp. 552-594.

Cardona, P. (2014): "Florentino González y la defensa de la República", Araucaria. Revista Iberoamericana de Filosofía, Política y Humanidades, 16(32), pp. 435-458

Collier, S. (2015): Chile. La construcción de una República, 1983-1865. Política e ideas, Santiago, PUC.

Condori, J. (2010): "Sociedad, identidad y regionalismo en Arequipa, 1780-1830", Historia, (9), pp. 47-71.

Covo, J. (1971): "L’image de Juárez dans la presse française à l'époque de l'intervention au Mexique (1862-1867)", Bulletin Hispanique, 73(3-4), pp. 371-395.

David, J. y David, T. (2011): "Vers une uniformisation culturelle ?", en Singaravélou, P. y Venayre, S. dirs., L'Histoire du monde au XIXe siècle, París, Fayard, pp. 209-221.

De Vega, M. (2001): dir., Historia de las relaciones internacionales de México, 18212010, v. 4, México, Secretaría de Relaciones Exteriores, 2011. 
Delli Quadri, R. (2014): "Latinoamericanos en Europa”, en De Francesco, A., Mascilli Migliorini, L. y Nocera, R., comps., Entre Mediterráneo y Atlántico. Circulaciones, conexiones y miradas, 1765-1867, Santiago, Fondo de Cultura Económica, pp. 437456.

Demélas, M-D. (1992): L’invention politique. Bolivie, Equateur, Pérou au XIXe siècle, Paris, Editions Recherche sur les Civilisations.

Duchesne, A. (1976): "Comentarios de la prensa internacional sobre la expedición belga a México", Estudios de Historia Moderna y Contemporánea de México, 5, pp. 93-108.

Feliú Cruz, G. (1933): En torno de Ricardo Palma, v. 1, Santiago, Prensas de la Universidad de Chile, p. 127-129.

Figueroa, P. P. (1900): Diccionario Biográfico de estranjeros en Chile, Santiago, Imprenta Moderna.

Galdámez, L. (1930): "Ramón Sotomayor Valdés", Anales de la Universidad de Chile, año 8, oct.-dic., serie 2, pp. 1565-1626.

García, D. (1992): "Los inicios del constitucionalismo peruano (1821-1842)", Pensamiento Constitucional, año IV, (4), pp. 233-244.

Gobat, M. (2013): "The Invention of Latin America: A Transnational History of AntiImperialism, Democracy, and Race”, American Historical Review, 118, pp. 1345-1375

Gootemberg, P. (1989): Between Silver and Guano. Commercial Policy and the State in Postindependence Peru, Princeton, Princeton University Press.

Gouttman, A. (2008): La Guerre du Mexique, 1862-1867. Le mirage américain de Napoléon III, Paris, Perrin.

Guerra, F.-X. (1989): "La lumières et ses reflets: Paris et la politique latinoaméricaine", en Kaspi, A. y Marès, M., dirs., Le Paris des étrangers, París, Imprimerie Nationale, pp. 172-181.

Gutiérrez, D. (2009): "Los primeros colombianos en París (1824-1830)", Anuario colombiano de historia social y de la cultura, 36 (1), pp. 89-124. 
Hale, C. (1957): "The War with the Unites States and the Crisis in Mexican Thoughout", The Americas, 14 (2), pp. 153-173.

Halperin, T. (2008): Historia contemporánea de América latina, Madrid, Alianza Editorial.

Henderson, P. (2008): Gabriel García Moreno and Conservative State Formation in the Andes, Texas, University of Texas Press.

Holguín, O. (1984): "Palma y Torres Caicedo: una amistad literaria", Fénix, 30-31, pp. 230-256.

Irigoin, M. (2010): "Gresham a caballo: las raíces monetarias de la fragmentación política de la América española en el siglo XIX", en Contreras, C., Mazzeo, C y Quiroz, F., comps, Guerra, finanzas y regiones en la historia económica del Perú, Lima, Banco Central de Reserva del Perú-IEP, pp. 219-269.

Juárez, B. (1972-1975): Documentos, discursos y correspondencia, México, Editorial Libros de México.

Kalifa, D., Régnier, P., Thérenty, M-E y Vaillant, A., (2011): dirs., La civilisation $d u$ journal. Histoire culturelle et littéraire de la presse en France au XIXe siècle, París, Nouveau Monde.

Lacoste, P. (1997): “Americanismo y guerra a través de El Mercurio de Valparaíso (1866-1868)", Anuario de Estudios Americanos, 54(2), pp. 567-591.

Lastarria, J. (1878): Recuerdos Literarios, Santiago, Imprenta de Jacinto Nuñez.

Lecaillon, J-F. (1994): Napoléon III et le Mexique : les illusions d'un grand dessein, París, Harmattan.

Lempérière, A. (2004): Entre Dieu et le Roi, la République. Mexico, XVIe - XIXe siècles, París, Les Belles Lettres.

Letelier J. (2016): "Entre la costa de Cobija y tierras altas. El tráfico arriero a inicios de la república boliviana", Diálogo Andino, (49), pp. 225-234.

López Beltrán, C. (2016): La Ruta de la Plata: De Potosí al Pacifico. Caminos, comercio y caravanas en los siglos XVI y XIX. La Paz, Plural. 
López C. (1969): "La intervención francesa y el imperio en la prensa uruguaya", Historia Mexicana, 9(2), pp. 248-281.

Lyon-Caen, J. (2011): "Lecteurs et lectures : les usages de la presse au XIXe siècle", en Kalifa, D., Régnier, P., Thérenty M-E y Vaillant, A., dirs., La civilisation du journal. Histoire culturelle et littéraire de la presse en France au XIXe siècle, París, Nouveau Monde, pp. 23-60.

Marx, K. (2002), Les luttes des classes en France. París, Gallimard.

Medzibrodsky, E. (1980): "Repercusión del «imperio» de Maximiliano y de la lucha independentista del pueblo mexicano en la prensa húngara contemporánea", Estudios Latinoamericanos, 6, pp. 155-168.

Meyer, J. (2014): "Las oposiciones francesas a la expédition du Mexique", en Palacios, G. y Pani, E., coords., El poder y la sangre. Guerra, Estado y Nación en la década de 1860. México, El Colegio de México, pp. 451-479.

Mignolo, W. (2005): The Idea of Latin America. Oxford, Blackwell Pub.

Milza, P. (2004): Napoléon III. París, Perrin.

Novak, F. (2005): Las relaciones entre el Perú y Francia, 1827-2004. Lima, Universidad Católica del Perú.

Núñez, A. (1878): Memorias sobre las relaciones diplomáticas de México con los Estados libres y soberanos de la América del Sur. México, Imprenta del Gobierno.

Osterhammel, J. (2014): The Transformation of the World. A Global History of the Nineteenth Century. Princeton y Londres, Princeton University Press.

Palacios G. y Pani, E. (2014): coords., El poder y la sangre. Guerra, Estado y Nación en la década de 1860. México, El Colegio de México.

Palti, E. (2005), "La transformación estructural de la esfera pública latinoamericana en el siglo XIX y el surgimiento del modelo proselitista de la opinión pública", en Casáus Arzú, M. y Ledesma, M., eds., Redes intelectuales y formación de las naciones en España y América latina 1890-1940. Madrid, Ediciones de la Universidad Autónoma de Madrid, pp. 23-38 
Palza, H. (2013): Aprendiendo a ser libres. Los avatares del Perú republicano tras la independencia. Lima, Grupo Gráfico del Piero.

Pani, E. (2001): Para mexicanizar el Segundo Imperio. El imaginario político de los imperialistas. México, El Colegio de México.

Pani, E. (2002): "Dreaming of a Mexican Empire: The Political Project of the Imperialistas", Hispanic American Historical Review, 82(1), pp. 1-31.

Parodi, D. y González S. (2014): comps., Las historias que nos unen. 21 relatos para la integración entre Perú y Chile. Lima, PUCP.

Portocarrero, F. (2009): "From Oligarchy to Bourgeoisie: The Economic Elite in Peru, 1916-1960", Entreprises et histoire, 54(1), pp. 155-177.

Real de Azúa, M. (1978): "La misión diplomática del peruano Manuel Corpancho: 1862-1863”, Historia Mexicana, 28(1), 1978, pp. 62-81.

Rosemblitt, J. (2018): "Los circuitos comerciales en el eje Tacna-Arica durante la temprana República peruana, 1840-1863. Una aproximación a los comerciantes intermedios", RIRA, 3 (2), pp. 203-242.

Salomon, N. (1975): Juárez en la conciencia francesa. 1861-1867. México, Secretaría de Relaciones Exteriores.

Sanhueza, C. (2007): "En busca de un lugar en el mundo: viajeros latinoamericanos en la Europa del siglo XIX", Estudos Ibero-Americanos, 32 (2), pp. 51-75.

Santa Cruz, E. (2010): La prensa chilena en el siglo XIX. Patricios, letrados, burgueses y plebeyos. Santiago, Universitaria.

Santana, A. y Guerra S. (2006): comps., Benito Juárez en América latina y el Caribe. México, Universidad Nacional Autónoma de México.

Schoonover, T. (1986): ed., The Mexican Lobby: Matías Romero in Washington, 18611867. Lexington, University of Kentucky Press. 
Slauter, W. (2012): "Le paragraphe mobile. Circulation et transformation des informations dans le monde atlantique du XVIIIe siècle", Annales. Histoire, Sciences Sociales, 67 (2), pp. 363-389.

Suárez-Piña, B., Virginia, C. y Durán-Rodríguez, G. (2016): "Los periódicos de Santiago de Cuba en el siglo XIX: reflexiones sobre su importancia patrimonial", Revista Santiago, Especial VLIR, pp. 114-124.

Subercaseaux, B. (2002): Historia del libro en Chile. Desde la colonia hasta el Bicentenario. Santiago, LOM.

Tamayo, F. (2017): “Autoritarismo y liberalismo. Una mirada a partir de la obra de Florentino González a la ideología liberal en Colombia en el siglo XIX", Estudios Políticos, (51), julio-diciembre, pp. 106-127.

Tauzin-Castellanos, I. (2015): dir., Histoire des itinéraires et des étapes en Amérique latine. XVIe-XIXe siècle. Bordeaux, Maison des Sciences de l'Homme d'Aquitaine.

Taylor, L. (1987): "Voluntarios extranjeros en los ejércitos liberales mexicanos, 18541867, Historia Mexicana, 37 (2), pp. 205-237.

Thibaud, C., (2017): Libérer le Nouveau Monde. La fondation des premières républiques hispaniques. Colombie et Venezuela (1780-1820). París, Les Perséides.

Uribe J. (1974): Tipos y cuadros de costumbres en la poesía popular del siglo XIX. Santiago, Mueller.

Vaillant, A. (2011): “L'Histoire au quotidien”, en Kalifa, D., Régnier, P., Thérenty, M-E y Vaillant, A., dirs., La civilisation du journal. Histoire culturelle et littéraire de la presse en France au XIXe siècle. París, Nouveau Monde, pp. 1319-1328.

Vaillant, A. (2017) : "Le règne de l'imprimé", en Singaravélou, P. y Venayre, S., dirs., Histoire du monde au XIXe siècle. Fayard, pp. 195-208.

Van Aken, M. (1989): King of the Night. Juan José Flores \& Ecuador. 1824-1864. California, University of California Press.

Villalobos, S. (2002): Chile y Perú. La historia que nos une y nos separa. 1533-1883. Santiago, Universitaria. 
Walker, C. (2001): “'La orgía periodística': prensa y cultura política en el Cuzco durante la joven República", Revista de Indias, 61 (221), pp. 7-26.

Zoraida, J. y Meyer, L. (2013): México frente a Estados Unidos. Un ensayo histórico, 1776-2000. México, Fondo de cultura Económica.

Fecha de recepción: 19 de diciembre de 2019.

Fecha de aceptación: 9 de enero de 2020. 